\title{
La expansión de una ciudad compacta: el caso de Huesca.
}

\author{
Elena Lacilla Larrodé \\ Departamento de Teoría, Proyectos y Urbanismo, \\ Escuela Técnica Superior de Arquitectura de la Universidad de Navarra, Pamplona, España \\ E-mail:mlacilla@unav.es
}

\begin{abstract}
Resumen. Las ciudades españolas han experimentado su mayor expansión urbana durante la segunda mitad del siglo XX. Este hecho fue debido a la migración campo-ciudad que tuvo lugar durante ese periodo. El modelo urbano que respondió a esa situación tuvo como marco legal la Ley del Suelo de 1956 que impulsaba el desarrollo por poligonos.

Así, la ciudad de Huesca pasó de 14.632 habitantes en 1940 a 50.085 en 1990, lo que significó un aumento en la demanda de vivienda. Esto llevó a una extensión del ámbito urbanizado que partía de $60 \mathrm{Ha}$. y que ha alcanzado las $550 \mathrm{Ha}$., contando con edificación que alberga tanto uso residencial como industrial -si bien la ocupación residencial es de $400 \mathrm{Ha}$. aproximadamente-

Sin embargo, a pesar de este aumento de ocupación, desde la administración se persiguió la intención de mantener una ciudad compacta. Desde el primer Plan General de Ordenación Urbana para Huesca, la solicitud por parte de los oscenses de un ámbito en la periferia de la ciudad para albergar viviendas unifamiliares no ha cesado. En una primera instancia (1958) se demandaron viviendas agrícolas, despues (1980) viviendas tipo chalet y más recientemente (1994) la diversidad de tipologías donde debian existir viviendas unifamiliares. Todas estas sugerencias, y esto es lo que se pretende exponer en este artículo, se han atendido e integrado en la expansión de la ciudad a través de Planes Parciales cubriendo las necesidades de los ciudadanos y evitando la difusión desmesurada de la ciudad en el territorio.
\end{abstract}

$$
\begin{gathered}
\text { Palabras clave: Huesca, ciudad compacta, expansión urbana, } \\
\text { variedad tipológica, vivienda unifamiliar, } \\
\text { siglo XX, planes parciales. }
\end{gathered}
$$

\section{Introducción}

En el año 1949, mediante una carta del arquitecto local José Urzola al ayuntamiento, comenzó a considerarse la necesidad de un Plan General de Ordenación Urbana, dado el crecimiento que se vislumbraba. Así en 1958 se aprobó el primer PGOU de Huesca bajo el marco legal de la Ley del Suelo de 1956. Hasta entonces la ciudad oscense había crecido paulatinamente y se habían desarrollado algunos núcleos urbanos mediante la tipología de vivienda unifamiliar. Estos grupos de viviendas unifamiliares que se construyeron en la primera mitad del siglo XX fueron: Villa Isabel con 64 viviendas cuyo proyecto se realizó en 1941 y se construyó en 1948, la Ciudad Jardín Ernesto Gil Sastre con 44 viviendas proyectado en 1954 y construido inmediatamente después, Estrecho Quinto con 72 viviendas proyectado en 1950 y construido a lo largo de esa década y el grupo Pamplona Blasco proyectado y construido durante la década de los '50 y que albergó 20 viviendas.

Imagen 1.

A excepción de este último todos los demás se ubicaron en la parte Oeste de la ciudad, sobre la planificación del segundo Ensanche. El emplazamiento sobre la retícula ya planeada del Ensanche garantizaba el control sobre la expansión de estos núcleos que, aunque se 


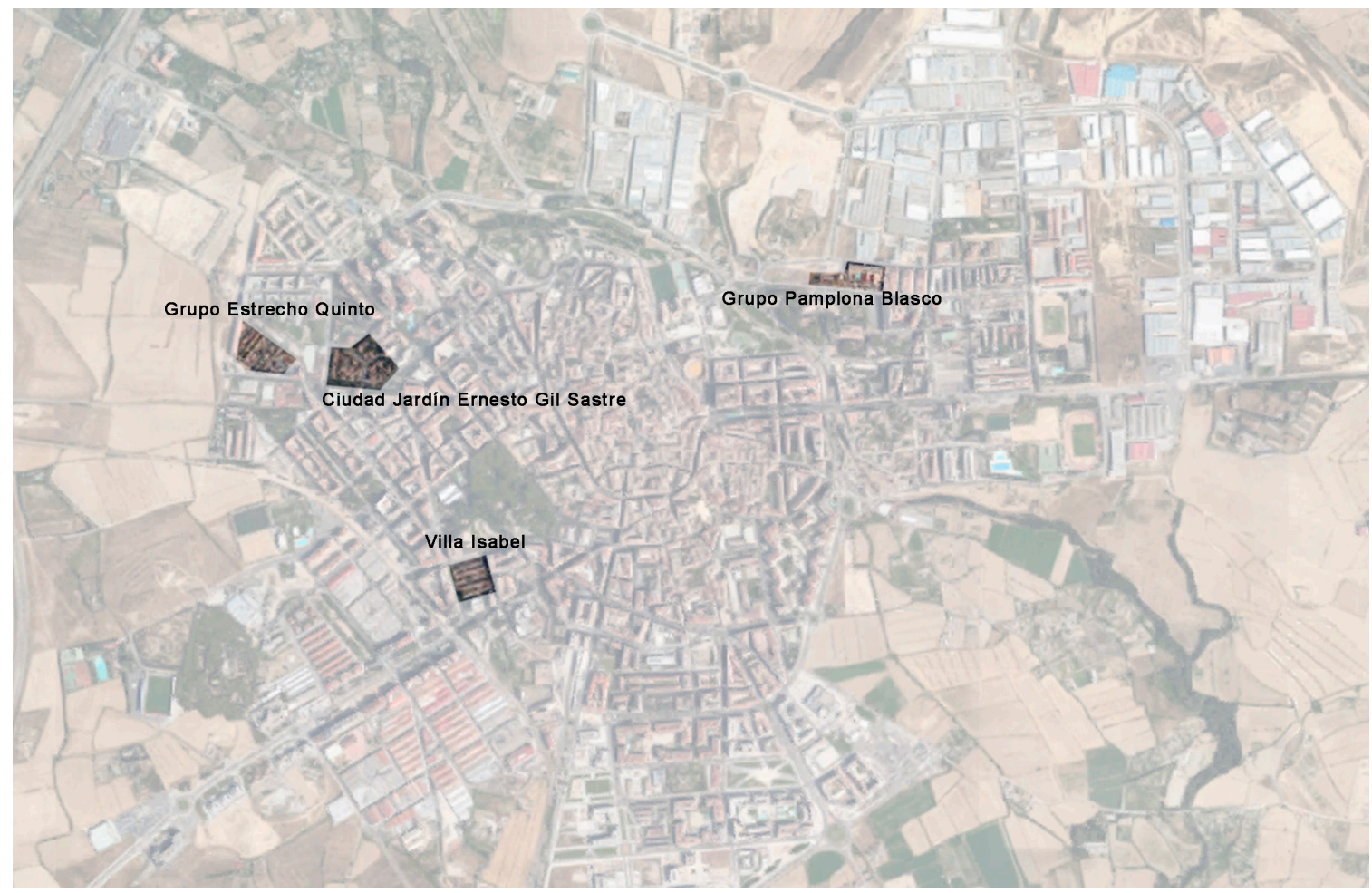

Fig. 1. Grupos de viviendas unifamiliares anteriores al Plan General. (Elaboración propia, 2019)

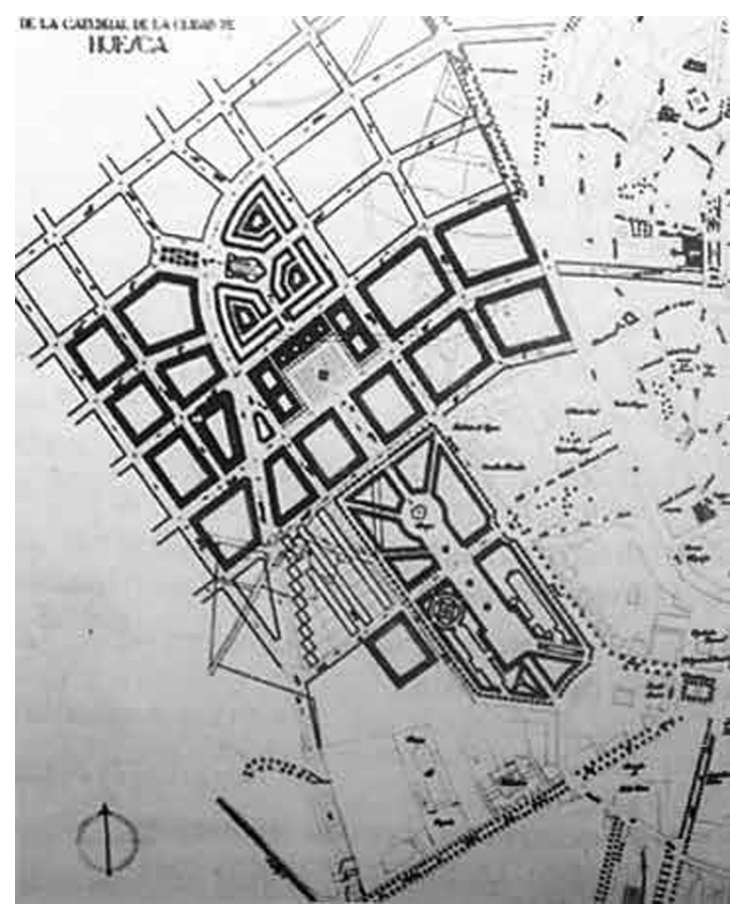

Fig. 2. Plano del Segundo Ensanche. (Archivo Municipal de Huesca, Miguel Aranda García, 1941)

ubicaron en la periferia del ámbito planificado, quedaba acotado a un número concreto de parcelas.

A partir de entonces, durante la segunda mitad del siglo XX, las ciudades españolas experimentaron su mayor expansión urbana. Este hecho fue debido a la migración campociudad que tuvo lugar durante ese periodo. El modelo urbano que respondió a esa situación tuvo como marco legal la Ley del Suelo de 1956 que impulsaba el desarrollo por polígonos. Asimismo, la ciudad de Huesca pasó de 14.632 habitantes en 1940 a 50.085 en 1990 , lo que significó un aumento en la demanda de vivienda. Esto llevó a una extensión del ámbito urbanizado que partía de $60 \mathrm{Ha}$. y que ha alcanzado las $550 \mathrm{Ha}$., contando con edificación que alberga tanto uso residencial como industrial -si bien la ocupación residencial es de $400 \mathrm{Ha}$. aproximadamente-.

Sin embargo, a pesar de este aumento de ocupación, desde la administración se persiguió la intención de mantener una ciudad compacta a pesar de que la demanda de viviendas unifamiliares fuera continuada. Asimismo, los planes generales fueron constantes en su idea de un crecimiento compacto de la ciudad y, aunque se intentó dar respuesta a esa demanda cada vez que se solicitaba -principalmente en los periodos de exposición pública de los Planes Generales-, hasta la actualidad ya no 
se ha vuelto a ejecutar ninguna agrupación exclusivamente dedicada a vivienda unifamiliar como había ocurrido anteriormente. A partir de entonces, la vivienda unifamiliar quedaría integrada junto con la vivienda colectiva en el diseño urbano que desarrollaría los polígonos residenciales.

En el primer Plan General de 1958, se presentó una alegación durante el periodo de exposición pública que interpuso la Hermandad Sindical de Labradores y Ganaderos en mayo de 1958, ya que en su momento "no se atendió a esta justa petición". Dicha alegación hacía referencia a unas viviendas agrícolas que querían ubicar en la parte Sur de la ciudad para sus afiliados, concretamente entre el camino viejo de Salas y de la Cabañera. Esos terrenos figuraban en el Plan como Zona Exterior, siendo considerados por la Hermandad como idóneos para construir una barriada agrícola urbana, tanto por su situación geográfica como por la dirección de los vientos. De esta forma se intentaba la desaparición de focos de olores en el centro de la ciudad y el paso obligado de carros y vehículos cargados por las principales vías. Esta petición llegó a buen puerto pues, las viviendas agrícolas contaron con su sector propio en el lugar donde se solicitaba, el Polígono 29 y cuya planificación fue desarrollada en 1960, como veremos.

Posteriormente, durante la revisión del primer PGOU, en 1980, también se sugirió reservar bolsas de suelo para destinarlas a la edificación de viviendas tipo chalet o vivienda aislada. Una sugerencia muy propia de Huesca ya que empezaba a ser constante y común en las alegaciones de mayoría de los organismos. Estos solicitaban la reserva de suelo para viviendas chalets o la creación de una zona destinada a la construcción de ciudad jardín, ya que si seguía creciendo el nivel de vida cada vez sería más acentuada la demanda de este tipo de construcciones. Debido a ello, en el desarrollo de los planes parciales 25 y 28 se reservaron dos áreas que albergarían viviendas del tipo demandado.

Asimismo, en el Avance del PGOU en 1994, también se consideró la necesidad de la variedad tipológica ya incluida en la memoria del documento. Y prueba de ello fue la aparición de viviendas unifamiliares en el planeamiento y ejecución del Polígono 24-II, también llamado Padre Querbes. Quizá este plan parcial es el que mejor integra esta tipología en el cojunto global del ámbito de desarrollo.

En base a estas observaciones se estudian a continuación y con más detalle las siguientes actuaciones: el Polígono 29 que, aunque no se llegó a ejecutar, responde a la demanda que tuvo lugar durante la exposición pública del PGOU de 1958; los Polígonos 25 y 28 que responden a las alegaciones de la exposición pública del PGOU de 1980; y el Polígono 24-II que albergó viviendas unifamiliares como respuesta al análisis realizado para la elaboración del avance del PGOU de 1994.

\section{Viviendas agrícolas. Polígono 29}

El Plan Parcial del Polígono 29 fue visado por el Colegio Oficial de Arquitectos de Aragón y Rioja en junio de 1960, aunque no se llegó a aprobar siendo el encargado de desarrollarlo el Arquitecto Miguel Aranda García. El polígono estaba destinado exclusivamente a viviendas de tipo agrícola, y éste sería el único emplazamiento de toda la ciudad donde se permitirían. Contaba con 180.000 metros cuadrados y el trazado urbano que se proyectó comprendía una serie de calles longitudinales y transversales de doce metros de anchura dando lugar a manzanas que permitían una parcelación adecuada al tipo de vivienda agrícola a que estaba reservado el polígono, viviendas que como es lógico debían tener corral amplio con anejos para cuadras, gallineros, cochiqueras, etc. Por eso, las ordenanzas de este plan parcial no permitían la ocupación de más del $40 \%$ de la superficie de la parcela. Dada la extensión de la zona que comprendía el polígono y su situación en un extremo del casco urbano, se consideró conveniente prever una plaza que al mismo tiempo que rompía la monotonía de las calles, comprendiera un pequeño centro cívico con iglesia, dependencias parroquiales, salón de actos y grupo escolar, situándose esta plaza en la zona Este de las dos en que se dividía el polígono por ser la más alejada del casco urbano. En el lado Oeste también se proyectaron dos plazas con jardines, con objeto de aminorar la densidad de la población (Aranda, 1960). 
Pero en aquel momento el Ayuntamiento carecía de medios económicos para proceder a la urbanización del polígono. El objetivo del plan parcial era fundamentalmente que los particulares pudiesen proceder a la realización parcial de estos servicios con objeto de tener una zona en que se pudiese edificar.

Según las ordenanzas, el carácter que hubiera tenido este sector de la ciudad si se hubiese llevado a cabo ${ }^{2}$, dado el momento en que se redactó, en 1960, parece que hubiese sido algo así como un Poblado Agrícola ${ }^{3}$. Las semejanzas con los Poblados Agrícolas podrían ser consecuencia del autor del plan parcial. Miguel Aranda García había trabajado para la Administración desde el momento en que acabó sus estudios de arquitecto lo que significa, casi con total seguridad, que conocía los asentamientos que se estaban llevando a cabo en España por parte de los organismos públicos, y entre los que estaban los Poblados Agrícolas destinados a la sociedad que hasta el momento habían vivido de la agricultura. Sin embargo, por las fechas, habrían sido un hecho inusual, pero ayudan a entender el carácter singular de la sociedad de una ciudad como Huesca en aquel momento.

Tanto el emplazamiento del Polígono 29 y el de los Poblados Agrícolas se encontraba en la periferia de la ciudad, consiguiendo abaratar el coste de los terrenos y mayores superficies de las parcelas, así como evitar las molestias de la maquinaria agrícola y los malos olores en el conglomerado urbano. Asimismo, la tipología planteada en los Poblados Agrícolas coincidía con la que se planteaba en Huesca, basada en viviendas con una cuadra donde podría caber un carro y una caballería, y un patio que servía de corral (Moya, 1983) 4 . Pero la diferencia fundamental era que el Polígono 29 estaba destinado a personas que ya vivían en Huesca y se trataba de una simple relocalización, mostrando el carácter rural de la ciudad incluso en la segunda mitad del Siglo $\mathrm{XX}^{5}$.

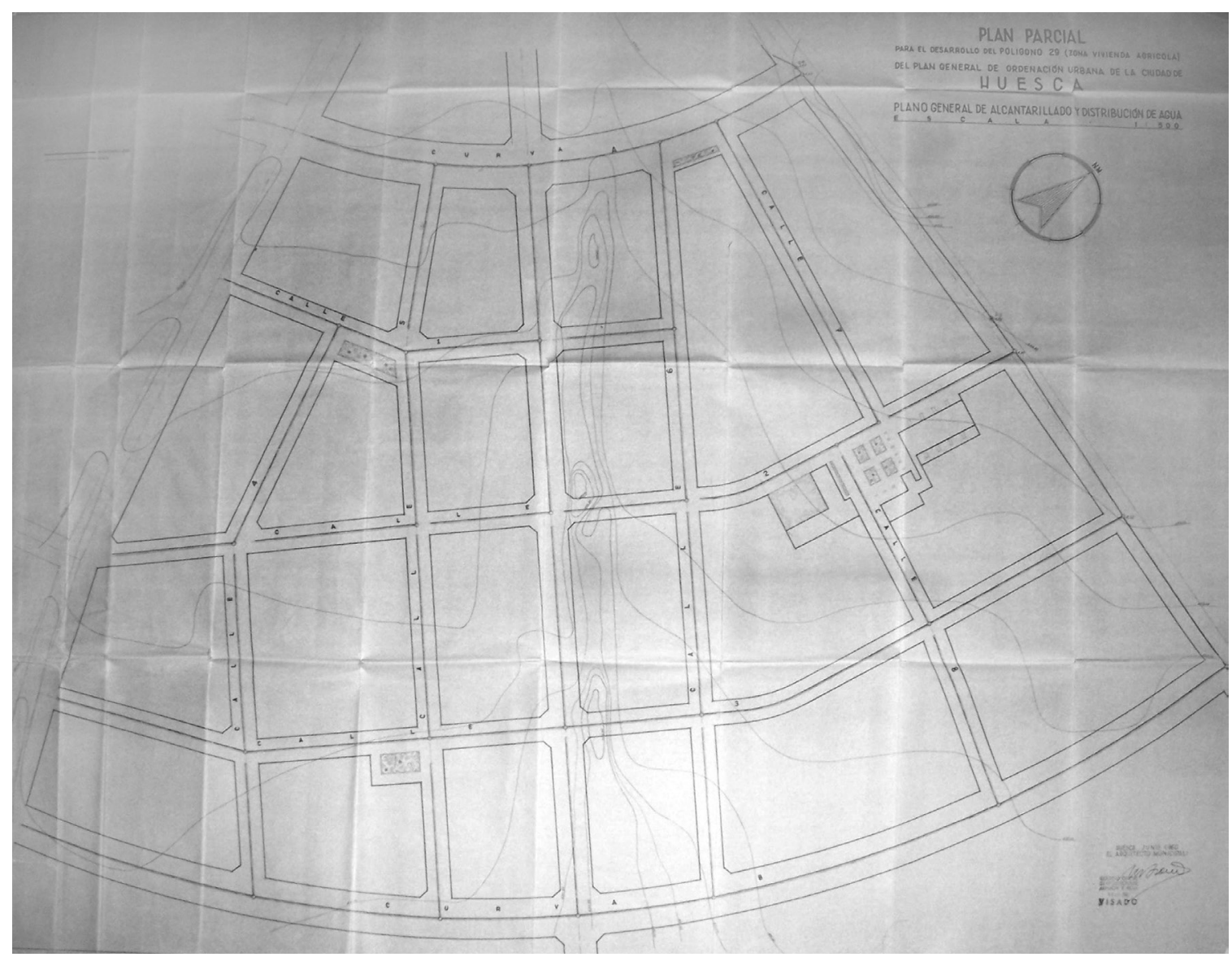

Fig. 3. Plano general de alcantarillado y distribución de agua del Polígono 29. (Archivo Municipal de Huesca, Miguel Aranda García, 1960). 


\section{Polígono 28}

El Polígono 28 o también denominado Polígono de Los Olivos tenía ya su delimitación contenida en el PGOU de $1958^{6}$. Sin embargo debido a ello y al escaso número de construcciones existentes, el Plan del 80 tuvo la oportunidad de proponer variaciones en la organización y zonificación existentes, cambiando la edificación cerrada por abierta, eliminando un elevado número de calles y aumentando las superficies destinadas a equipamientos. De este modo la ordenación global que estableció el Plan General se basó en la división del polígono en tres franjas longitudinales de Este a Oeste. Cada una de ellas contenía un uso predominante. Así, las dos bandas exteriores correspondían a la edificación residencial quedando la banda central reservada a espacio libre al mismo tiempo que se concentraban ahí todos los equipamientos. De esta forma se conseguía una estructura clara y ordenada que conectaba con la ciudad existente (Guzmán, 1982).

Ahora bien, el Plan General solo se limitó a determinar la estructura general y la delimitación de cada manzana siendo la ordenación pormenorizada dentro de ellas de carácter no vinculante. Por ese motivo se hacía necesario un estudio de detalle de cada una de las manzanas. Por ello, la descripción anterior de la ordenación del Plan General para este polígono es relevante, por el hecho de que el Estudio de Detalle de las manzanas A, B, C y D del Polígono 28 posterior aceptaría su planeamiento y partiría de las determinaciones descritas. Esto pudo ser debido a que dicho estudio se elaboró en 1981, sólo un año más tarde de la aprobación del PGOU y a que, además, los redactores de ambos documentos fueron los mismos: los arquitectos Guzmán Folgueras y Fernández Castro.

Por tanto, a la hora de desarrollar el estudio de detalle, según los autores, en primer lugar, fue necesario determinar la tipología edificatoria a emplear eligiendo el bloque en $\mathrm{H}$ pese a los inconvenientes que posteriormente se manifestaron. Lo más destacable de esta actuación fue la forma de agrupación de esta tipología ya que se aproximaba a la de manzana tradicional. Sin embargo, en la configuración resultante no llegaba a cerrarse la edificación en sí misma, como lo haría la manzana del ensanche decimonónico, sino que dejaba aberturas en ocasiones puntuales. Por
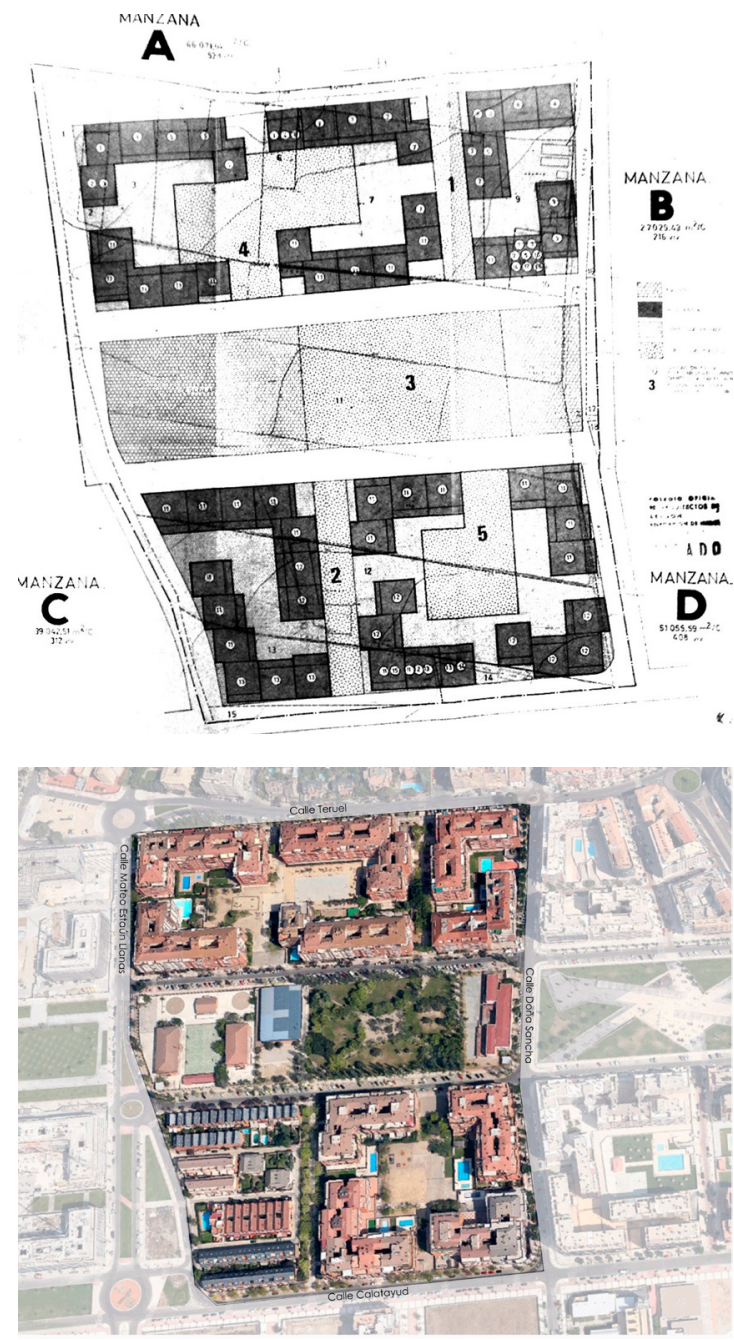

Fig. 4. (arriba) Plano de conjunto. Estudio de Detalle de las manzanas A, B, C y D del Polígono 28. (Archivo Municipal de Huesca, GUZMÁN FOLGUERAS, Manuel y FERNÁNDEZ CASTRO, Alfonso. 1982).

Fig. 5. (abajo) Ortofoto de estado actual del Polígono 28. (Elaboración propia, 2015).

tanto, dichos espacios interiores tendrían un carácter privado, pero sin acceso restringido. Por ello, el Polígono 28 fue clave dentro del significado del urbanismo oscense ya que representa el paso de una mentalidad antigua a una nueva a través de diferentes tipologías. $\mathrm{Su}$ diseño urbano se apoyaría principalmente en el tipo edificatorio con forma de $\mathrm{H}$ pero con la búsqueda de un tejido urbano basado en la manzana tradicional. Significó un caso de 
acercamiento a la recuperación de la manzana desde parámetros externos al nuevo paradigma.

Ahora bien, cabe destacar que una de las manzanas proyectadas en principio para albergar la misma tipología que el resto de la propuesta, finalmente se ejecutó mediante viviendas unifamiliares adosadas. Esta zona dio cabida a 93 viviendas unifamiliares, que a su vez rompía el lenguaje de manzana abierta que representaban el resto de las manzanas. Este hecho refleja la necesidad de cubrir esa demanda que venían solicitando los ciudadanos.

\section{Polígono 25}

El Polígono 25 suponía un vacío en la zona occidental de la ciudad ya que se encontraba próximo al ensanche Oeste con una importante densidad de población. Esto se debía a su calificación como zona de reserva ferroviaria durante la vigencia del PGOU de 1958 motivo por el que este ámbito no participó previamente del desarrollo urbano pese a su ubicación próxima a la zona del ensanche. Al mismo tiempo, el Polígono 25 era considerado como la oportunidad de generar dotaciones en el ámbito Oeste. Es precisamente este hecho lo que motivó el interés municipal por este plan parcial en el sentido de tomar la iniciativa del planeamiento por acuerdo plenario de fecha 21 de noviembre de 1980 y encargándose de su redacción ese mismo mes el arquitecto municipal Jesús Tejada aprobándose inicialmente el 9 de junio y definitivamente el 25 de octubre de 1983 (Tejada, 1982).

La trama adoptada se articulaba por el equipamiento escolar como rótula sirviendo el sistema de espacios libres, principalmente el gran boulevard central, de hilo conductor a modo de columna vertebral del polígono. La trama mantenía el carácter de retícula del ensanche próximo, pero con un tratamiento de la manzana radicalmente distinto a lo existente en el entorno. La manzana dejaba de ser cerrada para abrir sus espacios interiores y que se integraran en el sistema de espacios libres públicos del polígono. Así estos espacios posibilitaban recorridos peatonales alternativos y al mismo tiempo se potenciaban gracias a los accesos a las viviendas a través de estos
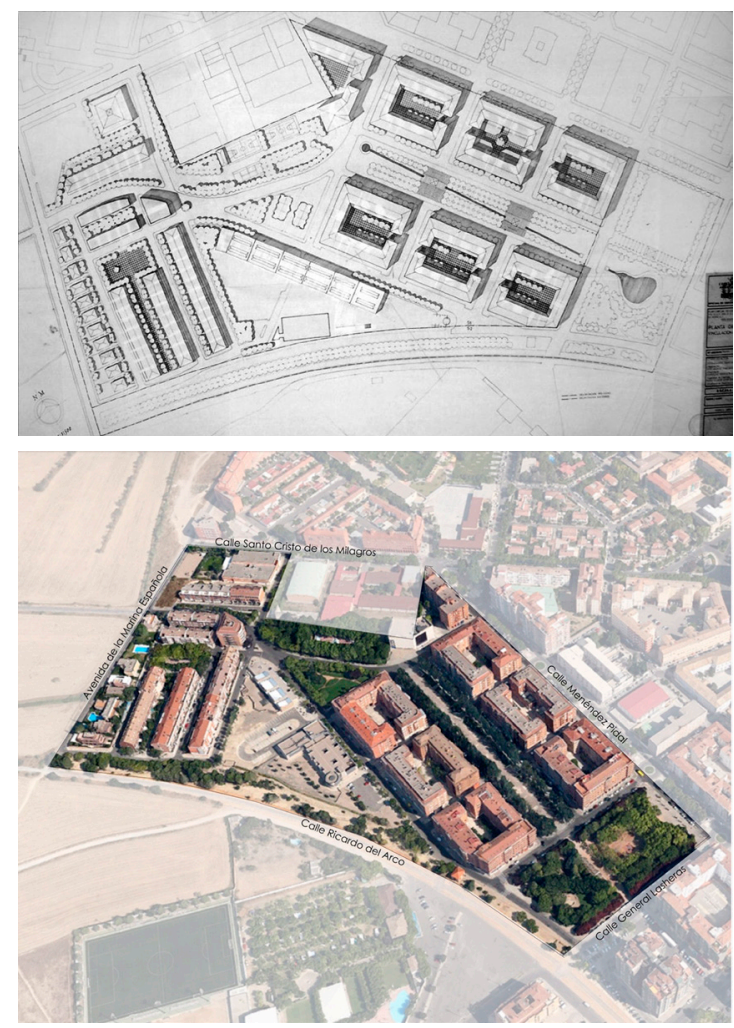

Fig. 6. (arriba) Plan Parcial de los Sectores 1 y 2 del Polígono 25. (Archivo Municipal de Huesca, TEJADA, Jesús. 1983).

Fig. 7. (abajo) Ortofoto de estado actual del Polígono 25. (Elaboración propia, 2015).

espacios. La tipología edificatoria se basaba en el bloque a dos fachadas y triple crujía. Se imponía la obligación de realizar soportales en la avenida de Menéndez Pidal, como elemento generador de la vida urbana de la que carecía totalmente esa vía urbana.

Pero lo más relevante fue la reserva de un área con una trama diferenciada respecto a las seis manzanas referidas anteriormente. Esta zona lindaba con el suelo urbanizable no programado, que en aquel momento era el campo, y se le daba un tratamiento de ciudad jardín con edificaciones de media y baja densidad que iban desde el bloque lineal de nueve metros de fondo hasta la vivienda unifamiliar en hilera o aislada, posibilitándose diversas soluciones en cada caso, de modo que la curva de densidades careciera de escalones producidos por un borde muy densificado y sin transición.

Esta zona, aunque situada en el borde del ámbito y en contacto con el suelo todavía sin 
urbanizar, fue capaz de dar cabida a la tipología de viviendas unifamiliares para, una vez más, cubrir esa demanda latente de los oscenses. En este caso, se contó con este ámbito desde el inicio, quedando la estructura de las seis manzanas totalmente completa a diferencia del Polígono anterior donde la tipología unifamiliar sustituyó uno de los agregados en forma de manzana que componía el conjunto.

\section{Polígono 24-II}

En el Polígono 24-II, contrariamente al mantenimiento de una tipología de ensanche (manzanas), representaría una evolución hacia una mayor libertad de diseño, dentro de los parámetros propios de la recomposición de la ciudad y de la atención a los tipos tradicionales. Es decir, conscientes de la necesidad de continuar la ciudad (trama, trazados, tipos) el diseño no se quedaría en una mera repetición de la manzana del ensanche.

La propuesta que se formulaba en esta modificación consistía en, siguiendo la filosofia del Avance del Plan General, plantear una intervención aislada orientada a recalificar una superficie que en aquel momento estaba ocupada por instalaciones docentes y deportivas de carácter privado, antiguas y obsoletas. A partir de ello se diseñaría una nueva área de edificación residencial y un parque público completado con los correspondientes viales, zonas peatonales y de aparcamiento. Todo ello en conexión con su entorno urbano más próximo estableciendo una coordinación futura en el borde de la ciudad.

El marcado carácter residencial del entorno determinaba la implantación del uso residencial en el terreno como solución más acorde con la realidad. Por tanto, apostaron desde el inicio por la elección de tipologías abiertas con viviendas unifamiliares con jardín privado, y colectivas en bloques abiertos con jardines comunitarios y jardines privados, y grandes espacios dotacionales públicos con el fin de conseguir la menor ocupación posible y conseguir que la mayor parte del suelo sobre el que se actuaba terminase constituyendo un parque público. Este parecía ser el habitar que solicitaba la mayor parte de la población y ese era el intento de ciudad que pretendían realizar los redactores.

Concretamente la edificación se concentró en la parte Norte del ámbito, dejando el gran parque público en la zona Sur. Se diseñó con la intención de que existiera una relación directa entre los espacios verdes privados de las parcelas residenciales y el parque mencionado. Con este mismo respeto hacia las zonas ajardinadas se había escalonado la edificación, de manera que los edificios más altos se situaban al Norte y los de menor altura al Sur, de forma que los jardines públicos y privados presentasen siempre un soleamiento adecuado.

Dentro del área ocupada por la edificación se distinguían tres franjas tipológicamente distintas (fig.III.35). En primer lugar, la franja situada al Norte fue constituida por edificación abierta en agrupación. Esta comprendía cinco parcelas, cada una de ellas compuesta por uno o dos bloques formando una $\mathrm{L}$ y dejando un espacio libre interior donde se situarían zonas de juegos, piscinas, etc. Esta edificación presentaba la mayor altura (planta baja más cuatro y ático). En segundo lugar, la franja intermedia constituida por edificación cerrada semi-intensiva en bloque. Estaba compuesta por cuatro parcelas en las que la edificación se escalonaba desde las cuatro alturas del frente con el vial central hasta las dos plantas en la fachada hacia el parque. La tipología de viviendas podía ser aquí muy variada, con viviendas en planta baja con jardines privados, pisos en plantas alzadas con grandes terrazas y dúplex en las plantas más altas. En cuanto al volumen, se cuidaba especialmente el diseño de las terrazas escalonadas hacia el parque que en la medida de lo posible presentarían una composición volumétrica clara y urbana, con intención de poner en relación directa las viviendas con el parque a través de las "bandejas" que configuraban las terrazas. Y la tercera franja constituida por edificación cerrada semi-intensiva en vivienda unifamiliar de edificación que ocupaba tres parcelas. Eran viviendas unifamiliares en una altura de planta baja más dos que se agrupaban adosándose por uno, por dos o por tres frentes. Se buscaba con estas viviendas una escala de edificación adecuada que hiciera que la agrupación se integrase en el parque de una manera natural. Los jardines privados serían espacios de 


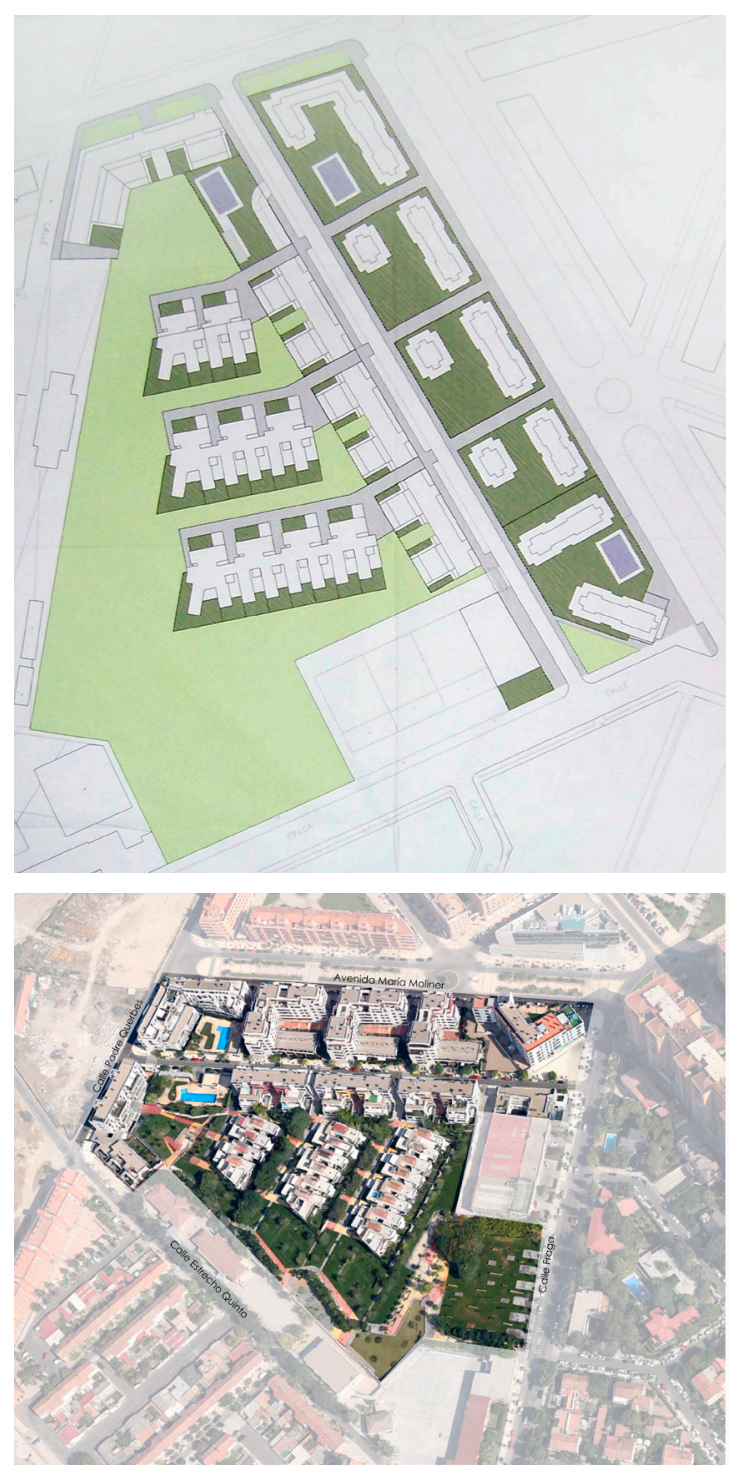

Fig. 8. (arriba) . Plano de ordenación del Polígono 24-II. (Archivo Municipal de Huesca, LACRUZ, Francisco y RUIZ MONSERRAT, Concepción. 2000). Fig. 9. (abajo) Ortofoto de estado actual del Polígono 24-II. (Elaboración propia, 2015).

transición entre las viviendas y el parque (Lacruz, 1999).

El gran parque del Polígono 24-II se funde con las viviendas en un intento de crear un diálogo entre ellos a través del paisaje. Este último significaría el punto máximo en cuanto a la atención al diseño del paisaje en la capital oscense. El detalle con el que se diseña y el criterio de sobrediseñar todo el espacio hasta el último metro cuadrado empezarían a ser comunes desde entonces a lo largo de toda la primera década del Siglo XXI.

\section{Conclusión}

Las cuatro propuestas estudiadas -P.29, P.25, P.28 y P.24-II- son las únicas que albergan la tipología de vivienda unifamiliar en Huesca desde que se aprobara el PGOU de 1958, y no lo hicieron de manera exclusiva como sí había ocurrido antes con otras agrupaciones de viviendas exclusivamente unifamiliar como se ha visto en la introducción -Villa Isabel, Ciudad Jardín Ernesto Gil Sastre, Estrecho Quinto o el Grupo Pamplona Blasco-.

El motivo de la integración de esta tipología en Polígonos residenciales donde se buscaba una mínima densidad, y por tanto la predominancia de la vivienda colectiva, fue la incesante demanda de residencia unifamiliar por parte de los oscenses. Esto llevo a combinar la vivienda unifamiliar con la vivienda colectiva que según el momento de desarrollo del Plan Parcial atendía a diversas corrientes de diseño urbano provenientes de Europa, como hemos visto.

Ahora bien, la integración de la residencia unifamiliar fue progresiva en el tiempo.

En primer lugar, el Polígono 29 fue una propuesta excepcional e interesante pero que, por fortuna, no se llegó a realizar. El motivo fue que los porcentajes de población activa fueron modificados por el cambio de estructura social y mientras aumentó la población en el sector industrial o de servicios disminuyó la agrícola que seguía viviendo en sus casas de labrador (Tesa, 1972). En caso contrario Huesca contaría con un sector de la población viviendo en una trama demasiado dispersa en una zona muy próxima al conglomerado urbano. Este hecho seguramente hubiese roto la coherencia y el concepto de unidad que caracterizan el urbanismo de Huesca.

En segundo lugar, el Plan Parcial del Polígono 28 buscaba ser íntegramente una propuesta contemporánea a las experiencias europeas que se estaban produciendo dentro de lo que quedó calificado como neorracionalismo que, en definitiva, volvía a recuperar las formas tradicionales de la ciudad. Este término comenzó a popularizarse a raíz de una crítica de Rikwert a la XV Triennale de Milán de 1973, momento de autopresentación del movimiento de la Tendenza. Nacido por la necesidad de una 




Fig. 10. Ubicación de los Polígonos de estudio. (Elaboración propia, 2019).

nueva razón de ser del urbanismo, buscaría nuevas bases racionales en que apoyar toda técnica. Esta base se centraría en la atención a lo histórico según diferentes niveles. En primer lugar, operaría en la tipología considerada como elemento edificatorio básico (Krier, 1981). En segundo lugar, operaría a nivel de las agrupaciones tipológicas: el empleo de la manzana principalmente. En tercer lugar, los espacios urbanos que caracterizarían las intervenciones serían, sobre todo, en la mayoría de los casos, una recuperación historicista neta, bien se concretasen en calles comerciales, en plazas o en todo un repertorio de formas urbanas históricas como el "circus", el "crescent", o la secuencia concatenada de espacios públicos monumentales (Ordeig, 2004). Por ese motivo, la vivienda unifamiliar en un inicio no encajaba con estas tesis y fue de manera posterior cuando se sustituyó una de las cuatro manzanas, prevista para seguir el diseño de las otras tres, por vivienda unifamiliar.

En tercer lugar, el Polígono 25 se convertiría en la muestra evidente del proceso planificador propio de esa nueva mentalidad que se vislumbraba ya de alguna manera en el Polígono 28 referido antes. Como en Huesca, las propuestas de ordenación de nuevas áreas residenciales en el resto de España en la década de los ochenta se apartarían radicalmente de las tendencias imperantes en los decenios anteriores y, al intentar evitar los males innegables que presentaban las actuaciones unitarias de los años sesenta y setenta debido a una interpretación parcial de los postulados funcionalistas, mirarían al pasado y apostarían por formalizar la ordenación de nuevos barrios mediante unas extensiones urbanas que se acercarían más a las que propugnaron los ensanches emblemáticos del pasado siglo (Trapero, 1995). Esta recuperación de una forma urbana clásica fue asumida y puesta en práctica en Huesca a través del Polígono 25. Y esta vez, quedó patente de manera completa, es decir, desde el inicio se reservó un área que se destinaría a vivienda unifamiliar de forma que no intercediese en el diseño de las seis manzanas que respondían a esa corriente de 
diseño que recuperaba la manzana tradicional.

Y por último, la mayor integración de la vivienda unifamiliar en el conjunto de la propuesta se consiguió en el Polígono 24-II. En este caso, sin dejar de atender a las corrientes del momento, el Polígono se plantea como si se hubiese fragmentado la representación que tenemos de la ciudad para después recomponerla en una nueva posibilidad, rompiendo con el sistema establecido que suponían tanto el ensanche anexo como las actuaciones apoyadas en la corriente del neorracionalismo. El arquitecto Paco Lacruz, plantea en este ámbito periférico de la ciudad una ordenación donde el espacio libre cobra especial protagonismo. De ese modo, Lacruz trazó unas líneas que servirían de referencia para todo el proyecto pero que no dialogan con el contexto inmediato. Debido a ello, se planteó una nueva forma de habitar donde la diversidad tipológica depende de la ubicación en relación al parque. La vivienda en altura se plantea más alejada del parque y la vivienda unifamiliar quedaba totalmente integrada en el mismo. Asimismo, la volumetría de la edificación no responde a unas líneas rectas y limpias, sino que contiene retranqueos que albergan distintos espacios para nuevos usos. Por todo ello, esta actuación podría identificarse sutilmente con la corriente teórica de diseño urbano denominada postestructuralismo la cual apostaba por un nuevo orden donde se rechazaba la ciudad convencional y las soluciones tradicionales o históricas.

En definitiva, la vivienda unifamiliar en Huesca no ha sido predominante en su desarrollo urbano evitando un crecimiento de la ciudad en mancha de aceite sobre el territorio. Como resultado, se ha mantenido una ciudad compacta, con todas las ventajas que ello supone según los actuales postulados que debe seguir la ciudad contemporánea para conseguir un desarrollo lo más sostenible posible. Bien es cierto, que la vivienda unifamiliar ha tenido su espacio dentro de esa expansión, pero como se ha visto, ha quedado integrado en el conjunto de la ciudad de diferentes formas, pero que no ha impedido situar a Huesca dentro de las corrientes de diseño urbano y consiguiendo una integración final donde se pasa de reservar una zona aislada para la vivienda unifamiliar a significar una variedad tipológica más en el Polígono 24-II donde se le otorga incluso un verdadero protagonismo dentro del conjunto.

\section{Notas}

1 Escrito del representante de los agricultores de la ciudad (y concejal) para exponer en Pleno Municipal, en 31 de mayo de 1958. Carpeta Policía Urbana, año 1949-1958, AMH.

2 La agricultura española llevaba muchos años olvidada por los diversos regímenes políticos que habían gobernado el país durante el Siglo XIX y casi el primer tercio del XX. Además, la aparición de la crisis económica en los primeros treinta años del siglo pasado no hizo más que agravar la desfavorable situación del campo español. Por ese motivo, se construyeron casi trescientos pueblos de colonización dispersos por el amplio territorio español.

3 Los Poblados Agrícolas fomentaban la agricultura para que, todos aquellos que emigraban del campo a la ciudad pudiesen mantener su vida agrícola y el cambio a la ciudad no fuese tan brusco.

4 En Madrid, los Poblados Agrícolas, cuya concepción era más ideológica que real, fueron ocupados en parte por traperos que aún utilizaban la cuadra, y en la mayoría de los casos ésta fue transformada en otra habitación casi desde un principio.

5 De hecho, como ya se ha señalado, Emilio Larrodera quiso disimular y superar esta cualidad rural de Huesca a través del Plan General dándole a Huesca un ambiente más urbano y distinguido.

6 En el Plan General de 1958 este polígono estaba clasificado como urbano, con una organización estructural de edificación cerrada, con manzanas residenciales destinadas a vivienda agrícola, a excepción de tres de ellas dedicadas a zona verde, escuelas y comercio.

\section{Referencias}

Aranda García, Miguel. 1960. Memoria del Plan Parcial para el desarrollo del Polígono 29 (zona vivienda agrícola) del Plan General 
de Ordenación Urbana de la ciudad de Huesca. Huesca: Archivo de la Oficina Técnica de Huesca, Carpeta ${ }^{\circ} 18$, junio.

Centellas Soler, Vicente. 2010. Los pueblos de colonización de Fernández del Amo. Arte, arquitectura y urbanismo. Barcelona: Edición Fundación Caja de Arquitectos.

Moya González, Luis, Ezquiaga, José María e Inglés Musoles, Fernando. 1983. Barrios de promoción oficial Madrid 1939-1976. Madrid: Editorial Colegio Oficial de Arquitectos de Madrid.

Guzmán Folgueras, Manuel y Fernández Castro, Alfonso. 1982. Memoria del Estudio de Detalle del Polígono 28. Huesca: Archivo de la Oficina Técnica de Huesca, Carpeta diversos 329/1982. 357/81.

Tejada, Jesús. 1982. Memoria del Plan Parcial de los Sectores 1 y 2 del Polígono 25. Huesca: Archivo de la Oficina Técnica de Huesca, Número de Archivo 111, expediente número de tramitación 1077/80, diversos, año 1982/ número326. Estante 6, carpeta 111, ejemplar número 8 , archivo planos percha.

Lacruz, Francisco y Ruiz Monserrat, Concepción. 1999. Memoria de la Modificación Aislada del PGOU en el Polígono 24-II. Huesca: Archivo de la Oficina Técnica de Huesca, Carpeta 408, ejemplar número 1 , AOTH.

Tesa Ayala, Luis. 1972. Carta que envía al Ayuntamiento en el plazo de sugerencias. Huesca: Archivo Municipal de Huesca, Carpeta Plan General de Ordenación. Proyecto de Revisión y Adaptación. Diversos 1982, $\mathrm{n}^{\mathrm{o}} 313$, (legajo $2^{\circ}$ de dos) XIII, Sugerencias.

Krier, Robert. 1981. El espacio urbano. Barcelona: Editorial Gustavo Gili.

Ordeig Corsini, José María. 2004. Diseño urbano y pensamiento contemporáneo. Barcelona: Ed. Monsa.

Trapero, Juan Jesús. 1995. "Prólogo". En Los nuevos ensanches de Madrid. La morfología residencial de la periferia reciente, 19851993, 12. Madrid: Edita la Gerencia Municipal de Urbanismo del Ayuntamiento de Madrid. 